\title{
Sirtuin 1 suppresses mitochondrial dysfunction of ischemic mouse livers in a mitofusin 2-dependent manner
}

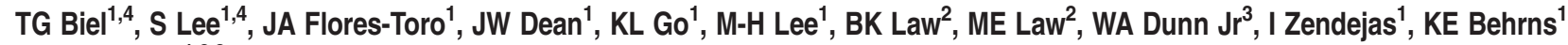 \\ and J-S Kim ${ }^{*, 1,2,3}$
}

Ischemia/reperfusion (I/R) injury is a major cause of morbidity and mortality after liver surgery. The role of Sirtuin 1 (SIRT1) in hepatic I/R injury remains elusive. Using human and mouse livers, we investigated the effects of I/R on hepatocellular SIRT1. SIRT1 expression was significantly decreased after I/R. Genetic overexpression or pharmacological activation of SIRT1 markedly suppressed defective autophagy, onset of the mitochondrial permeability transition, and hepatocyte death after I/R, whereas SIRT1-null hepatocytes exhibited increased sensitivity to I/R injury. Biochemical approaches revealed that SIRT1 interacts with mitofusin-2 (MFN2). Furthermore, MFN2, but not MFN1, was deacetylated by SIRT1. Moreover, SIRT1 overexpression substantially increased autophagy in wild-type cells, but not in MFN2-deficient cells. Thus, our results demonstrate that the loss of SIRT1 causes a sequential chain of defective autophagy, mitochondrial dysfunction, and hepatocyte death after I/R.

Cell Death and Differentiation (2016) 23, 279-290; doi:10.1038/cdd.2015.96; published online 17 July 2015

During hepatic resection and liver transplantation operations, inflow occlusion is employed to temporarily limit blood flow to minimize intraoperative blood loss. Although prolonged ischemia eventually causes tissue injury, severe damage paradoxically does not occur until recovery of blood flow and restitutions of normal physiological $\mathrm{pH} .{ }^{1}$ Ischemia/reperfusion $(\mathrm{I} / \mathrm{R})$ injury is a key cause of postoperative liver failure during hemorrhagic shock, hepatectomy, and liver transplantation. Despite continuous efforts, substantial benefits from current strategies have not been realized, mainly because of the multifactorial nature of I/R injury.

I/R initiates opening of high-conductance permeability transition pores in the mitochondrial inner membranes, leading to mitochondrial permeability transition (MPT). ${ }^{2}$ Onset of the MPT uncouples oxidative phosphorylation and depolarizes mitochondrial membrane potential $\left(\Delta \Psi_{\mathrm{m}}\right)$ that in turn causes ATP depletion and cell death.

Autophagy is an evolutionarily conserved catabolic process. Among the three forms of autophagy, macroautophagy is of particular importance in the liver, as it not only degrades unneeded intracellular proteins but also digests injured or dysfunctional organelles such as abnormal mitochondria. ${ }^{3}$ We have shown that impaired autophagy contributes to liver I/R injury. ${ }^{4-6}$

Sirtuin1 (SIRT1) deacetylates Lys residues of both histone and nonhistone targets, and is activated in response to fasting and calorie restriction in the liver, a condition inducing autophagy. ${ }^{7,8}$ Despite its extramitochondrial localization,
SIRT1 appears to affect mitochondrial biogenesis ${ }^{9}$ and bioenergetics, ${ }^{10}$ but its mechanisms remain elusive.

Using isolated hepatocytes, mouse livers, SIRT1-null mice, and human livers, we here demonstrate that I/R depletes livers of SIRT1 and that specific overexpression of SIRT1 mitigates defective autophagy, onset of the MPT, and subsequent hepatocyte death after both in vitro and in vivo $\mathrm{I} / \mathrm{R}$. Furthermore, we show that mitofusin-2 (MFN2) is a new substrate for SIRT1.

\section{Results}

SIRT1 is lost after I/R in human and mouse livers. To investigate the changes in SIRT1 after ischemia, three independent human liver biopsies were collected before and during a single episode of inflow occlusion (Figure 1a). Immunoblotting analysis showed that ischemia alone decreased SIRT1 by $\sim 70 \%$. Similar to human livers, in vivo ischemia to mouse livers markedly decreased SIRT1 to $27 \%$ of basal levels, and this was not recovered by reperfusion (Figure 1b).

Hepatocyte injury is the cardinal mechanism underlying liver dysfunction after warm I/R. ${ }^{1}$ Mouse hepatocytes were exposed to various periods of simulated ischemia. As shown in Figure 1c, ischemia alone significantly decreased SIRT1 to $43 \%$ of the control values after $2 \mathrm{~h}$ of ischemia. After the extension of ischemia to $4 \mathrm{~h}$, SIRT1 was virtually undetectable.

\footnotetext{
${ }^{1}$ Department of Surgery, University of Florida, Gainesville, FL, USA; 'Department of Pharmacology and Therapeutics, University of Florida, Gainesville, FL, USA and ${ }^{3}$ Department of Anatomy and Cell Biology, University of Florida, Gainesville, FL, USA

*Corresponding author: J-S Kim, Department of Surgery, University of Florida, R4-204 ARB, 1600 SW Archer Road, Gainesville, FL 32610 USA. Tel: +1 3523927461 ; Fax: +1 352265 1060; E-mail: Jae.Kim@surgery.ufl.edu

${ }^{4}$ These authors contributed equally to this work.

Abbreviations: AdGFP, adenovirus encoding green fluorescence protein; AdSIRT1, adenovirus encoding sirtuin 1; Akt, AKT8 virus oncogene cellular homolog; ALLM, acetyl-Leu-Leu-Met; CHX, cycloheximide; CQ, chloroquine; GTPase, guanosine triphosphatase; IP, immunoprecipitation; I/R, ischemia/reperfusion; KRH, Krebs-RingerHEPES; KO, knockout; LC3, microtubule-associated protein 1 light chain 3 protein; MEF, mouse embryonic fibroblasts; MFN, mitofusin; MPT, mitochondrial permeability transition; PI, propidium iodide; Rd-123, rhodamine-123; RSV, resveratrol; shMFN2, small hairpin RNA targeting MFN2; SIRT1, sirtuin 1; TMRM, tetramethylrhodamine methylester; VDAC, voltage-dependent anion channel; WT, wild type

Received 29.10.14; revised 22.5.15; accepted 03.6.15; Edited by N Chandel; published online 17.7.15
} 
a
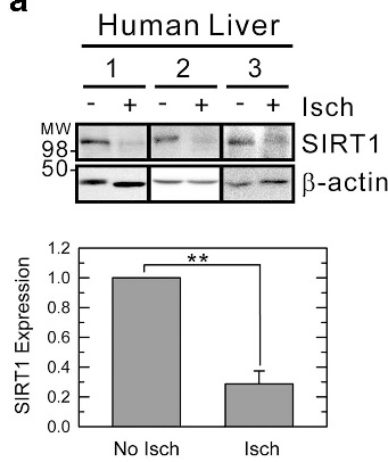

d

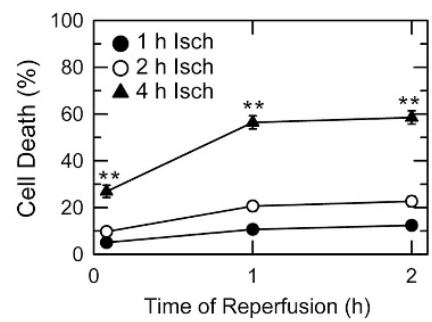

b
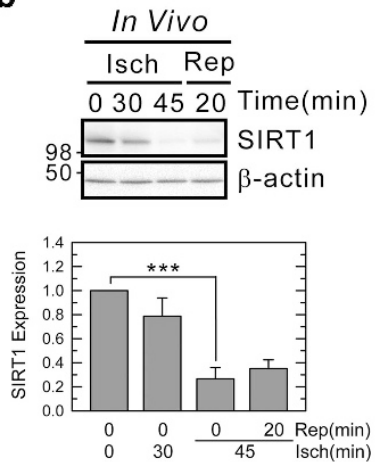

e

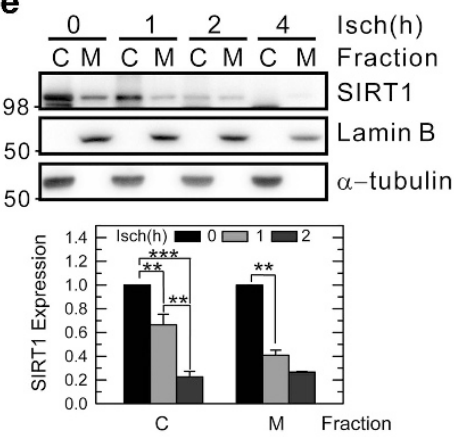

C
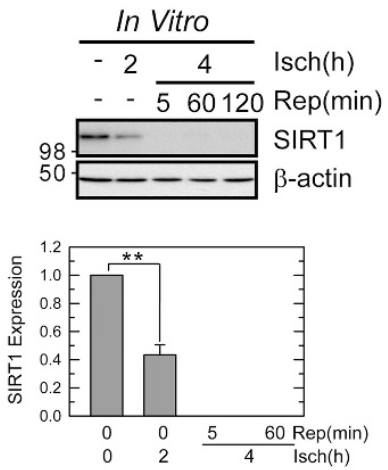

f

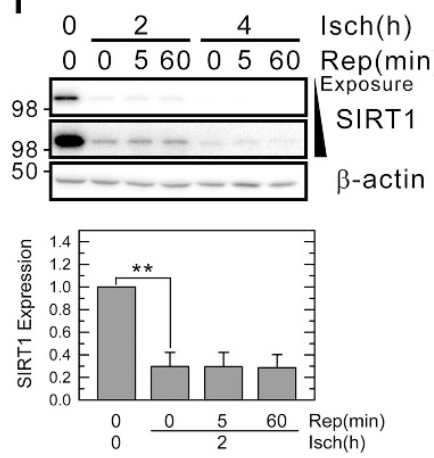

Figure 1 I/R causes SIRT1 loss. (a) Human liver tissue was collected before and during inflow occlusion and SIRT1 was immunoblotted. SIRT1 expression was normalized against $\beta$-actin and its expression relative to the value before ischemia was determined. (b) Mouse livers and (c) isolated mouse hepatocytes were subjected to in vivo and in vitro $I / R$, respectively, and SIRT1 was immunoblotted $(n=3)$. SIRT1 levels were normalized against $\beta$-actin and its expression relative to the value at 0 min of ischemia was determined. (d) Hepatocytes were subjected to various periods of ischemia and necrosis was measured at 5,60 , and 120 min after reperfusion $(n=5)$. ${ }^{* \star} P<0.01$ versus $1 \mathrm{~h}$ ischemia. (e) Hepatocytes were subfractionated into the cytosolic (C) and membrane (M) fraction during ischemia, and SIRT1 levels were immunoblotted ( $n=3)$. (f) After l/R, the changes in SIRT1 of hepatocytes were determined by immunoblotting $(n=3) .{ }^{* \star} P<0.01$, and ${ }^{\star \star \star} P<0.005$

Collectively, these data demonstrate that SIRT1 is prone to ischemia and that prolonged $\mathrm{I} / \mathrm{R}$ depletes hepatocytes of SIRT1.

Hepatocytes are resistant to a short-term ischemia. Cell death occurs only after reperfusion following prolonged ischemia (Figure 1d). To explore a potential correlation between the duration of ischemia, SIRT1 levels, and cell death, hepatocytes were subjected to different times of ischemia and the changes in SIRT1 were determined with immunoblotting (Figure 1e). Subcellular fractionation revealed that both cytosolic and membrane SIRT1 declined with ischemia. After $4 \mathrm{~h}$ of ischemia, a near-complete loss was observed, demonstrating a depletion of SIRT1 in both compartments by prolonged ischemia. Reperfusion failed to recover SIRT1 loss (Figure 1f), showing that reperfusioninduced cell death accompanies SIRT1 depletion.

SIRT1 overexpression suppresses the MPT after I/R. To investigate whether SIRT1 loss is causatively linked to I/R injury, we overexpressed SIRT1 with an adenoviral vector (AdSIRT1; Figure 2a). Adenovirus encoding a green fluorescent protein (AdGFP) was used as a viral control. The hepatocytes overexpressing SIRT1 significantly suppressed cell death after I/R. As MPT is a causal event in I/R-mediated liver injury, ${ }^{2}$ we next examined whether SIRT1 overexpression can prevent the onset of MPT. The opening of permeability transition pores, $\Delta \Psi_{\mathrm{m}}$, and cell death were simultaneously monitored using laser scanning confocal microscopy of calcein, tetramethylrhodamine methylester (TMRM), and propidium iodide (PI), respectively (Figure $2 \mathrm{~b}$ ). ${ }^{4}$ Briefly, MPT pores of the mitochondria remain closed in normal hepatocytes, leading to the exclusion of green fluorescing calcein by the mitochondria. Hence, normal mitochondria in the calcein images appear as dark and round voids wherein each void represents a single, polarized mitochondrion that takes up TMRM. However, once MPT pores open, calcein diffuses into the mitochondria and TMRM is simultaneously released from the mitochondria. After $4 \mathrm{~h}$ of ischemia, TMRM fluorescence was hardly detectable in both control and SIRT1-overexpressed hepatocytes because of anoxic depolarization. Calcein, however, exclusively labeled the extramitochondrial compartments, indicating a lack of MPT onset during ischemia. Within 3 min of reperfusion, the mitochondria in control hepatocytes transiently repolarized, but depolarized later (top panels). Concomitantly, calcein began to fill the mitochondria, denoting the MPT onset. Both calcein and TMRM fluorescence vanished at $23 \mathrm{~min}$ and cell death occurred, as visualized by nuclear labeling of $\mathrm{PI}$ (Figure 2b, arrows). In clear contrast, SIRT1-overexpressed hepatocytes continuously excluded calcein from the mitochondria and sustained both $\Delta \Psi_{\mathrm{m}}$ and viability (Figure 2b, bottom panels), indicating that SIRT1 overexpression prevents MPT onset, mitochondrial depolarization, and necrosis after I/R. Electron micrographs also exhibited the 


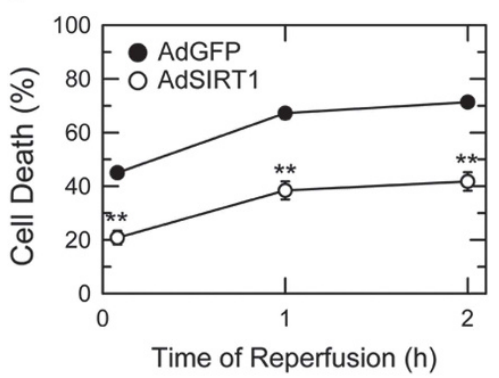

d

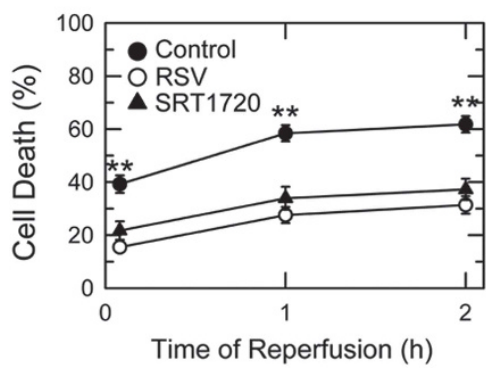

b

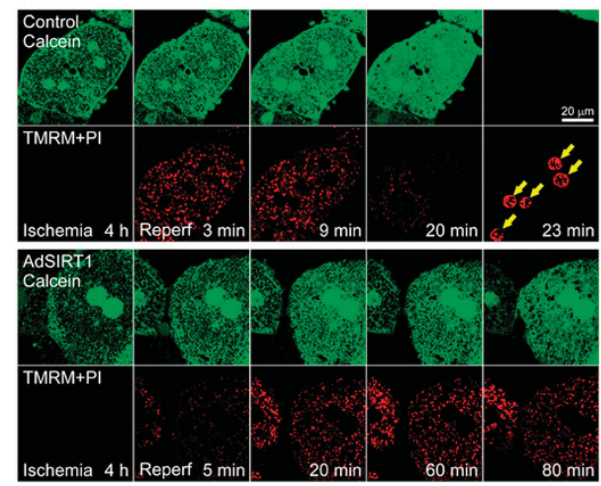

e

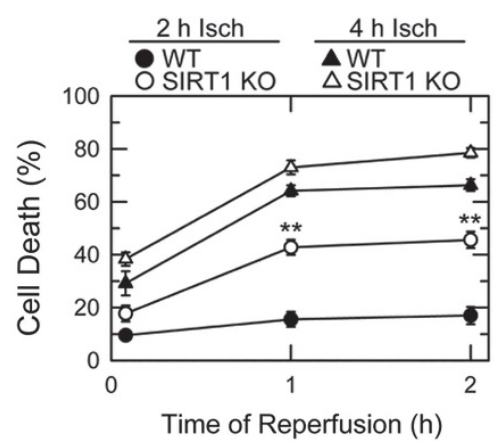

c

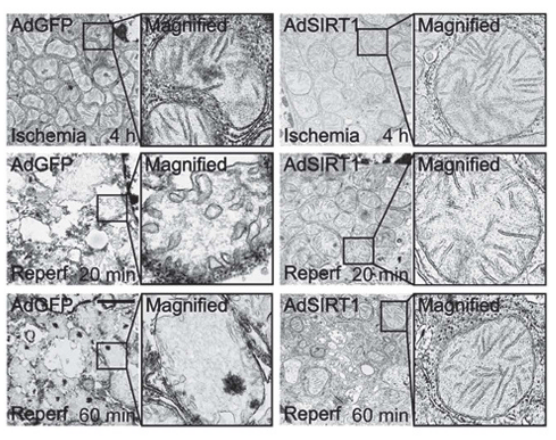

f

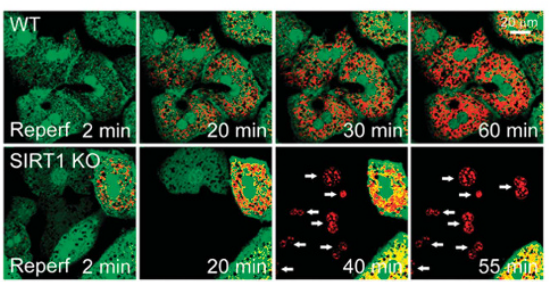

Figure 2 SIRT1 overexpression prevents MPTonset and necrosis after I/R in hepatocytes. (a) Hepatocytes were infected with AdGFP or AdSIRT1 (10 multiplicity of infection (MOI)), and exposed to $4 \mathrm{~h}$ of ischemia. Reperfusion-induced cell death was measured at 5, 60, and 120 min after reperfusion $(n=3)$. ${ }^{*} P<0.01$. (b) Confocal images were collected with and without SIRT1 overexpression to evaluate MPT, $\Delta \Psi_{m}$, and necrosis after I/R. (c) Electron micrographs of the mitochondria with and without SIRT1 overexpression during I/R. Right panels represent magnified images of square inserts in left panels. Scale bar: $2 \mathrm{~mm}$. (d) Cells were treated with resveratrol (RSV, $0.1 \mu \mathrm{M}$ ) or SRT1720 $(0.1 \mu \mathrm{M})$ for 16 or $1 \mathrm{~h}$, respectively, before ischemia. After $4 \mathrm{~h}$ of ischemia, reperfusion-induced cell death was measured $(n=3)$. ${ }^{* *} P<0.01$ versus RSV or SRT1720. (e) Cells from SIRT1 WT and KO mice were subjected to 2 or $4 \mathrm{~h}$ of ischemia and cell death was assessed after reperfusion $(n=3) .{ }^{*} P<0.01$ versus WT after $2 \mathrm{~h}$ ischemia. (f) MPT onset and cell death (arrows) of WT and KO hepatocytes were visualized with confocal microscopy of TMRM, calcein, and PI after $2 \mathrm{~h}$ of ischemia

maintenance of mitochondrial structural integrity by SIRT1 overexpression (Figure 2c). Thus, SIRT1 overexpression prevents MPT and mitochondrial defects, a causative mechanism of hepatocyte I/R injury. Pharmacological activation of SIRT1 was also cytoprotective (Figure 2d). When hepatocytes were treated with either resveratrol (RSV) or SRT1720, the SIRT1 activators, ${ }^{11}$ reperfusion-induced cell death was markedly thwarted.

To further clarify an integral role of SIRT1 in I/R injury, hepatocytes were isolated from tamoxifen-inducible SIRT1 conditional knockout mice (SIRT1 KO) (a gift from Dr. Sinclair, Harvard Medical School, Boston, MA, USA) ${ }^{12}$ and subjected to different periods of $\mathrm{I} / \mathrm{R}$ (Figure $2 \mathrm{e}$ and Supplementary Figure S1a). Although the hepatocytes from wild-type (WT) mice tolerated $2 \mathrm{~h}$ of ischemia and reperfusion well, the cells from SIRT1 KO mice displayed a profound injury (Figure 2e), showing increased sensitivity of SIRT1-null cells to a shortterm I/R. Reperfusion-induced death after $4 \mathrm{~h}$ of ischemia was, however, indiscernible between WT and KO cells. This observation was indeed anticipated as WT cells were eventually devoid of SIRT1 after $4 \mathrm{~h}$ of ischemia (Figure 1). Confocal imaging analysis also confirmed a rapid MPT onset and depolarization after $2 \mathrm{~h}$ of ischemia in SIRT1 KO cells (Figure 2f). Interestingly, the mitochondria of SIRT1 KO cells maintained $\Delta \Psi_{\mathrm{m}}$ and displayed no signs of MPT under normoxia (Supplementary Figure S1b). Hence, our results from biochemical, genetic, pharmacological, and imaging approaches support the hypothesis that the loss of SIRT1 contributes to mitochondrial dysfunction and hepatocyte injury after I/R.

SIRT1 depletion is calpain dependent. To delineate the mechanisms behind SIRT1 depletion, the changes in SIRT1 mRNA were measured with PCR (Figure 3a). I/R did not alter SIRT1 mRNA levels, suggesting that SIRT1 depletion is a post-translational process. Furthermore, immunoblotting after treatment with the protein synthesis inhibitor, cycloheximide $(\mathrm{CHX})$, estimated that the half-life of SIRT1 was $\sim 14 \mathrm{~h}$ (Figure 3b). Thus, SIRT1 loss is less likely associated with its chemical stability or turnover. Although SIRT1 overexpression was cytoprotective, the decline in SIRT1 protein was persistent after prolonged I/R (Figure 3c), further supporting the presence of an intrinsic pathway for SIRT1 degradation. Pharmacological activators also failed to block reperfusioninduced loss of SIRT1 (Supplementary Figures S2a and b). To identify the factor(s) involved in SIRT1 depletion, immunoblotting analysis was performed with calpain, cathepsin, and proteasome inhibitors. Calpain activation induces hepatic I/R injury. ${ }^{4-6}$ Acetyl-Leu-Leu-Met (ALLM), a specific calpain inhibitor, ${ }^{4,5}$ significantly suppressed the loss of SIRT1 (Figure 3d) and I/R injury (Figure 3e), suggesting that calpain is, at least in part, responsible for SIRT1 depletion. 
a

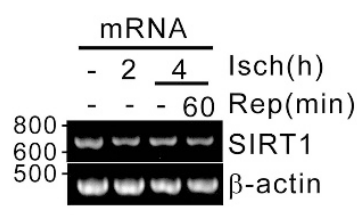

d

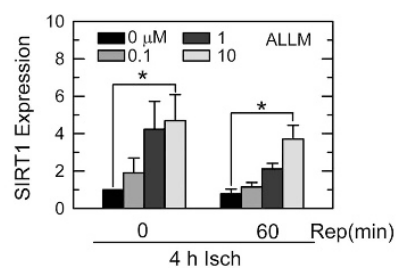

$-\frac{2^{4 h i s c h}}{-2-2}-\frac{4}{-2-606}$

- - - - - - $60606060 \operatorname{Rep}(\min )$

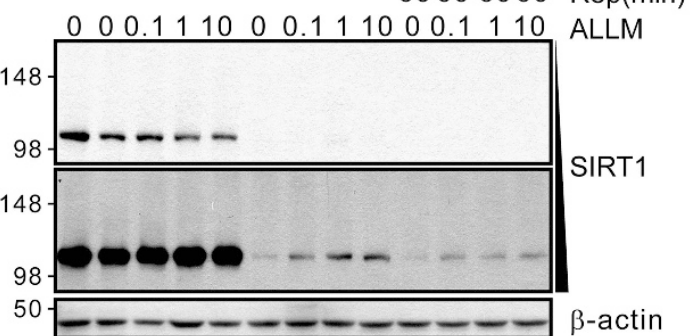

b
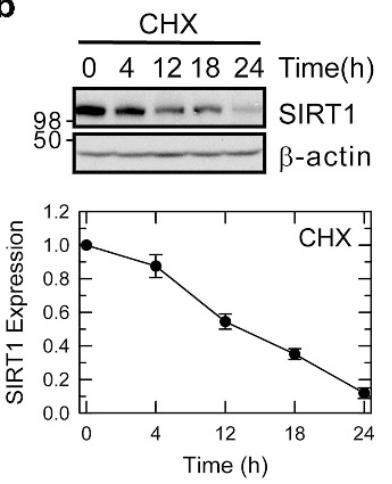

c

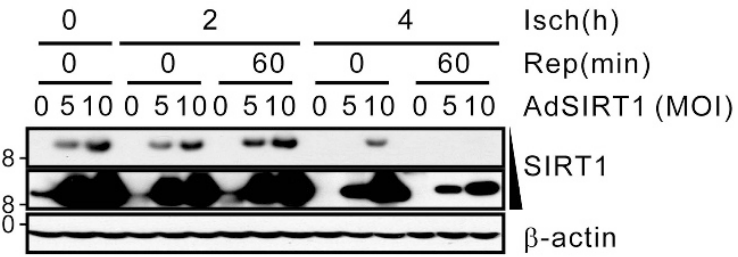

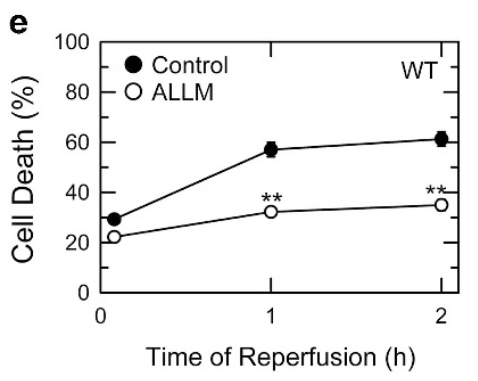

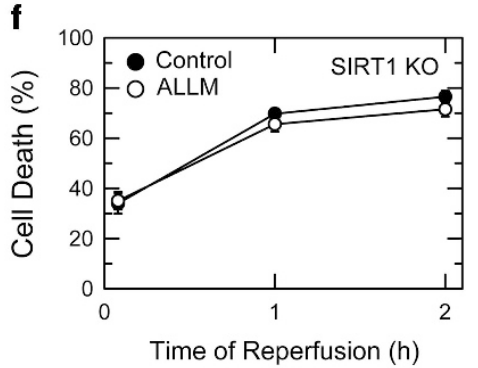

Figure 3 Calpain inhibition suppresses SIRT1 loss in hepatocytes. (a) The changes in SIRT1 mRNA were evaluated with RT-PCR. (b) Normoxic cells were treated with $35 \mu \mathrm{M}$ of cycloheximide (CHX) for up to $24 \mathrm{~h}$ and SIRT1 was immunoblotted $(n=3)$. SIRT1 levels were normalized against $\beta$-actin and its expression relative to the value at $0 \mathrm{~h}$ was determined. (c) SIRT1 expression after I/R was assessed at various titers of AdSIRT1. (d) Cells were treated with ALLM for $16 \mathrm{~h}$ before ischemia. After I/R, SIRT1 was immunoblotted. The changes in SIRT1 relative to the level at $0 \mathrm{~h}$ of reperfusion were plotted after normalization against $\beta$-actin $(n=3)$. Necrosis in (e) WT and (f) SIRT1 KO cells with and without $10 \mu \mathrm{M}$ of ALLM was measured after $4 \mathrm{~h}$ of ischemia $(n=3) .{ }^{*} P<0.05$ and ${ }^{*} P<0.01$

Fractionation experiments indicated that ALLM suppressed the loss of cytosolic SIRT1 after reperfusion, but not membrane SIRT1, implying the importance of cytosolic SIRT1 in hepatocyte viability (Supplementary Figure S2c). A crucial role of calpains was also confirmed in SIRT1 KO hepatocytes (Figure 3f). Although ALLM prevented reperfusion-induced death in WT hepatocytes, this agent did not suppress necrosis in KO cells. In other experiments, MG-132, a proteasomal inhibitor, neither suppressed SIRT1 depletion nor cell death after reperfusion (Supplementary Figures S3a-c). Cathepsins have been reported to degrade SIRT1 in endothelial cells. ${ }^{13}$ However, E64d, a cathepsin inhibitor, only marginally suppressed SIRT1 reduction after $2 \mathrm{~h}$ of ischemia (Supplementary Figure S3d). After $4 \mathrm{~h}$ of ischemia, E64d did not affect either SIRT1 levels or cell death (Supplementary Figures S3d-f). Thus, the roles for proteasomes or cathepsins in SIRT1 depletion are likely minimal. Of note, SIRT1 depletion and necrosis were slightly improved by simultaneous administration of both ALLM and E64d. Taken together, these results suggest involvement of calpains in SIRT1 depletion during I/R.

SIRT1 induces autophagy. Autophagy clears unnecessary or dysfunctional proteins and organelles in a lysosomedependent manner. Impaired autophagy contributes to $\mathrm{I} / \mathrm{R}$ injury to livers. ${ }^{4-6}$ To test whether SIRT1-mediated cytoprotection is associated with autophagy, hepatocytes were subjected to $\mathrm{I} / \mathrm{R}$ with and without SIRT1 overexpression, and autophagic flux was then assessed (Figure 4a).
Autophagic flux reflects the formation of autophagosomes and the clearance by autolysosomes, and truly represents the dynamic nature of autophagy. ${ }^{14}$ We determined autophagic flux by comparing the changes in microtubule-associated protein 1 light chain (LC3) with and without administration of chloroquine (CQ), a lysosomal inhibitor. Conversion of cytosolic LC3-I into autophagosomal LC3-II is indicative of autophagy induction. In control hepatocytes, autophagic flux after reperfusion was almost absent, consistent with previous reports. ${ }^{4-6}$ However, in the hepatocytes overexpressing SIRT1, a considerable autophagic flux was observed after I/R. The basal levels of LC3-II after SIRT1 overexpression were also significantly higher than the control, illustrating that SIRT1 not only prevents reperfusion-induced impairment of autophagy but also promotes basal autophagy. Increased autophagy by SIRT1 was further verified with both fluorescence and electron microscopy. Imaging analysis of GFP-LC3 labeled-hepatocytes displayed that SIRT1overexpressed cells encompassed numerous green puncta, representative of autophagosomes, whereas control cells had few autophagosomes and diffused GFP-LC3 fluorescence (Figure 4b). Furthermore, confocal imaging revealed that with SIRT1 overexpression, a subset of red fluorescing mitochondria became enwrapped by green fluorescing autophagosomes (arrow heads), signifying the onset of mitochondrial autophagy (mitophagy). Enhanced autophagy was also evident in electron micrographs, where SIRT1overexpressed cells exhibited multiple autophagic vesicles (Figure 4d, arrowheads) and more structurally intact 
a

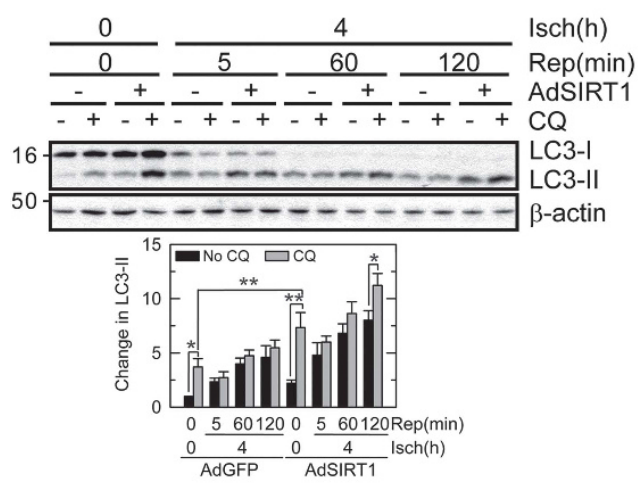

\section{b}

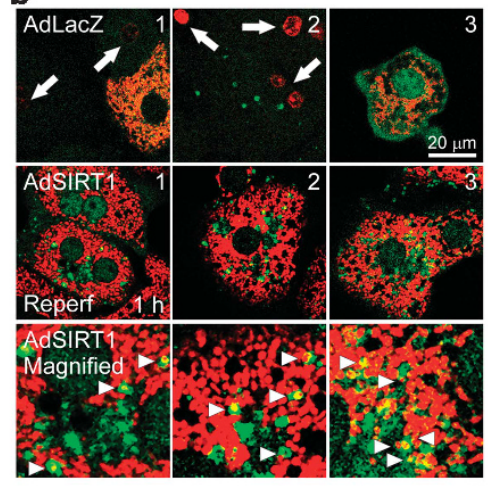

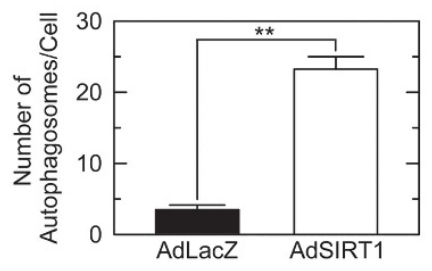

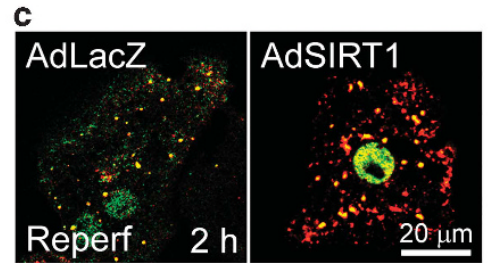

d

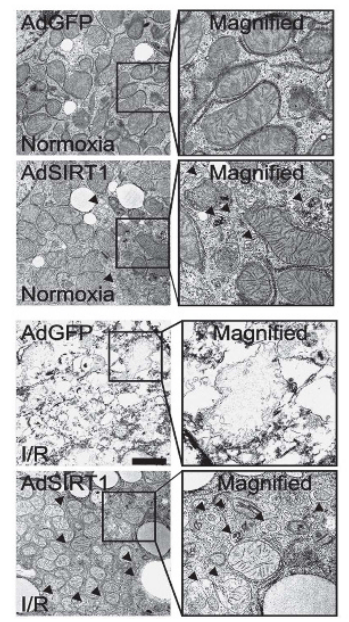

e

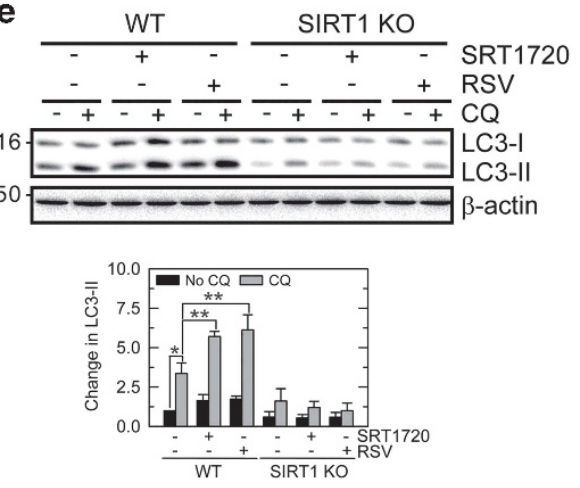

f
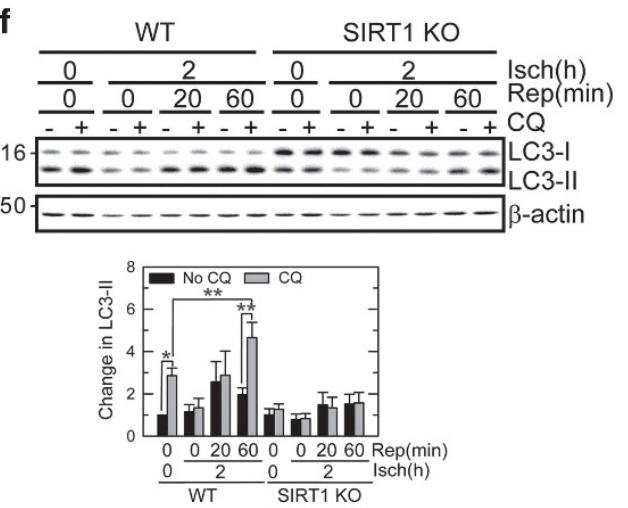

Figure 4 SIRT1 induces autophagy. (a) Hepatocytes were subjected to I/R with and without chloroquine (CQ) to determine autophagic flux ( $n=4)$. The expression of LC3-II relative to the level at $0 \mathrm{~h}$ of ischemia (no CQ) was determined after normalization against $\beta$-actin. (b) After $4 \mathrm{~h}$ of ischemia, confocal images of GFP-LC3, TMRM, and PI were collected in three different cells at $1 \mathrm{~h}$ of reperfusion. Note cell death (arrows) in control cells and the onset of mitophagy (arrowheads) in the SIRT1-overexpressed cell. The number of autophagosomes was counted per cell after 20 images were randomly collected per analysis. (c) Confocal images of a tandem mCherry-GFP-LC3 after $4 \mathrm{~h}$ of ischemia. (d) Electron micrographs of hepatocytes during normoxia (top panels) and after $1 \mathrm{~h}$ of reperfusion following $4 \mathrm{~h}$ of ischemia (bottom panels). Arrowheads indicate autophagic vesicles. Scale bar: $2 \mu \mathrm{m}$. (e) WT and SIRT1 KO cells were treated with RSV or SRT1720 under normoxia, and autophagic flux was determined ( $n=3$ ). The expression of LC3-II relative to the level of no treatment in WT was determined. (f) Autophagic flux after $2 \mathrm{~h}$ of ischemia was evaluated $(n=3)$. The changes in LC3-II were plotted relative to the level at $0 \mathrm{~h}$ of ischemia in $\mathrm{WT}$. ${ }^{\star} P<0.05$ and ${ }^{*} P<0.01$

mitochondria after I/R. We visualized autophagic flux in live hepatocytes with a tandem mCherry-GFP-LC3 probe. ${ }^{15}$ GFP loses its fluorescence in the acidic environment of autolysosomes, whereas the red fluorescence of mCherry is retained. Consequently, autophagosomes and autolysosomes emerge as yellow and red puncta, respectively. Confocal imaging showed a vast number of red puncta in SIRT1-overexpressed cells after $2 \mathrm{~h}$ of reperfusion (Figure 4c), confirming a stout autophagic clearance by SIRT1. Contrarily, few red puncta were visible in control cells.

The importance of SIRT1 in autophagy was further substantiated in SIRT1 KO hepatocytes. In WT cells, SIRT1 activators significantly increased basal autophagic flux (Figure 4e). However, neither activator enhanced autophagy in SIRT1 KO cells. Similarly, SIRT1 KO hepatocytes failed to sustain autophagic flux during a short-term I/R, a sublethal stress that does not impair autophagy in WT cells (Figure 4f). In addition, CQ did not increase normoxic LC3-II levels in SIRT1-null cells, corroborating the necessity of SIRT1 in basal autophagy. Altogether, these results support an essential role of SIRT1 in hepatocellular autophagy and survival after I/R.

Mitofusin 2 is a substrate for SIRT1. We next investigated how SIRT1 increases autophagy. Immunoblotting analysis of different autophagy and lysosomal proteins demonstrated a significant increase in cytosolic ATG7 expression under normoxia after SIRT1 overexpression (Figure $5 a$ and Supplementary Figure S4b). After I/R, no significant changes were, however, observed by SIRT1 overexpression (Supplementary Figure S4a). Moreover, SIRT1 overexpression neither inhibited mTOR nor promoted the expression of 5'-AMP-activated protein kinase or Akt (AKT8 virus oncogene cellular homolog) activation (Supplementary Figures S4c and d).

SIRT1 can deacetylate nonhistone proteins. ${ }^{16}$ Protein modification through acetylation/deacetylation regulates the mitochondria. ${ }^{17}$ To determine the changes in the acetylation status by SIRT1, immunoblotting was conducted with an acetylated Lys-specific antibody. Despite lack of noticeable 

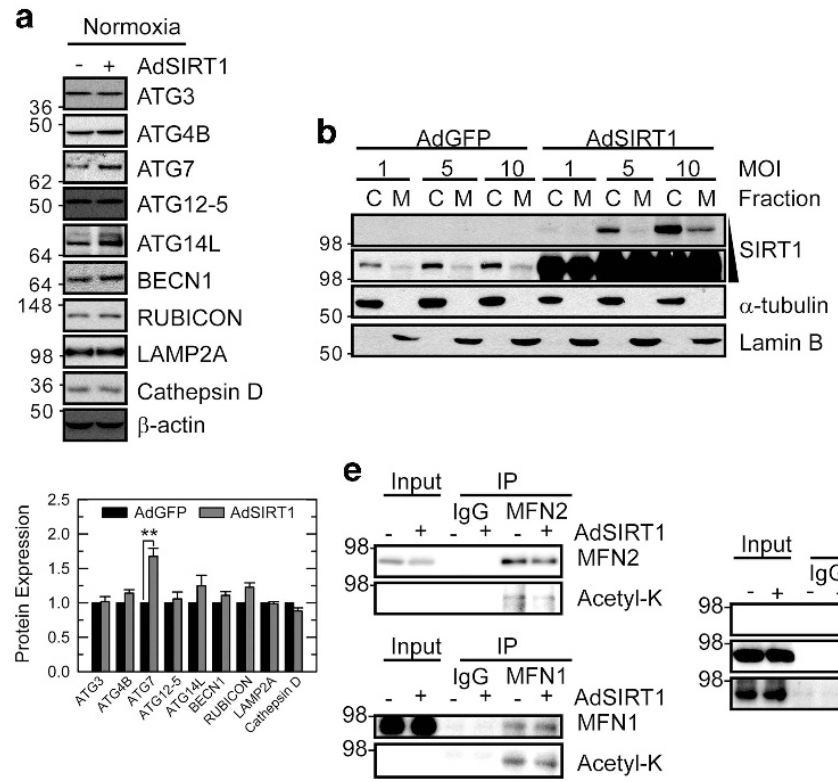

C

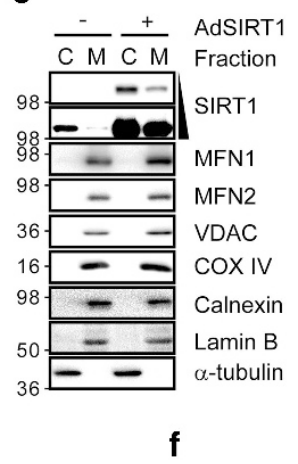

d

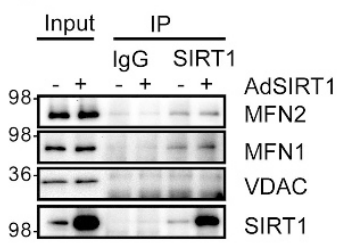

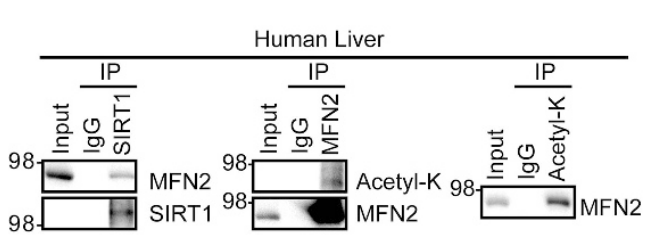

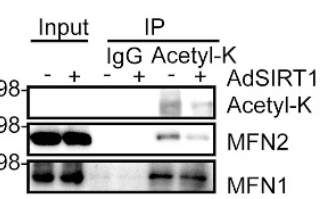

98

Figure 5 SIRT1 interacts with MFN2. (a) Protein expression before and after SIRT1 overexpression. ${ }^{* \star} P<0.01$. (b) Normoxic hepatocytes with different titers of AdSIRT1 were subfractionated into the cytosolic (C) and membrane (M) fraction, and SIRT1 was immunoblotted. (c) Immunoblotting of subcellular fractions with SIRT1, cytosolic ( $\alpha$-tubulin), mitochondrial (MFN1, MFN2, VDAC COX IV), endoplasmic reticulum (calnexin), and nuclear (Lamin B) markers. (d) The membrane fraction was immunoprecipitated (IP) with SIRT1 and subsequently immunoblotted with MFN or VDAC. (e) The membrane fraction was IP with MFN or acetyl-K, and immunoblotting with acetyl-K or MFN antibody, respectively. (f) IP and immunoblotting of human liver extracts

changes in whole-cell lysates (Supplementary Figure S4e), subcellular fractionation showed a substantial accumulation of SIRT1 in the cytosolic as well as membrane fraction (Figure 5b). Furthermore, SIRT1 overexpression appears to deacetylate some proteins in the membrane fraction containing the mitochondria (Figure $5 \mathrm{c}$ ), suggesting a mitochondrial substrate(s) for SIRT1. The acetylation status of FOXO1, FOXO3A, and PGC-1a was not altered after SIRT1 overexpression (Supplementary Figure S4f). Accordingly, we reasoned that SIRT1 could interact with the mitochondria, especially through outer membrane proteins such as mitofusin 1 and 2 (MFN1 and MFN2) and voltage-dependent anion channel (VDAC). The membrane fraction was prepared before and after SIRT1 overexpression, and immunoprecipitation (IP) was performed with SIRT1 antibody (Figure 5d). Immunoblotting after IP demonstrated a physical interaction of SIRT1 with MFN1 and MFN2, but not with VDAC.

We next tested whether SIRT1 can deacetylate either MFN1 or MFN2 or both. The combination of pull-down and immunoblotting approaches unveiled that both endogenous MFN1 and 2 existed as highly acetylated forms (Figure 5e). Importantly, SIRT1 overexpression markedly reduced the acetylated MFN2 levels, but not MFN1, suggesting that SIRT1 deacetylates MFN2. Like mouse livers, acetylated MFN2 existed endogenously in human livers and SIRT1 interacted with MFN2 (Figure 5f).

The N-terminal domains of MFN2 are necessary for SIRT1-dependent autophagy. MFN2 is a multidomain protein containing a GTPase (residues 98-260) and a transmembrane domain (residues 604-647), as well as two coiled-coil regions (residues 394-434 and $695-738) .{ }^{18}$ To investigate how SIRT1 interacts with MFN2, we constructed two deletion mutants of MYC-tagged MFN2; $\triangle 2-92$ and $\triangle 262-392$. The expression of these constructs in HEK293T cells was confirmed by immunoblotting (Figure 6a). Similar to hepatocytes, SIRT1 overexpression significantly increased autophagic flux in WT cells (Figure 6b). However, this increase was not observed in either mutant, and this was also confirmed by GFP-LC3 imaging analysis (Figure 6c), suggesting that two areas adjacent to the $\mathrm{N}$-terminal guanosine triphosphatase (GTPase) domain are critical in SIRT1-mediated autophagy induction.

To examine whether MFN2 deacetylation could change its catalytic activity, we collected confocal images of mitochondrial morphology in mouse embryonic fibroblasts (MEFs). MFN2 is a GTPase-directed mitochondrial fusion protein. ${ }^{19}$ Although WT cells have tubular and elongated mitochondria (data not shown), most mitochondria of MFN2 KO cells had fragmented morphology (Figure 6f). Reintroduction of MFN2 into the KO cells markedly rescued mitochondrial morphology. When SIRT1 was coadministered with MFN2, elongated mitochondria were still evident. The number of elongated mitochondria in the coadministered group was similar to that in the MFN2 alone group, inferring a comparable GTPase activity between two groups. Like hepatocytes, pull-down experiments also showed the interaction of SIRT1 with MFN2 as well as deacetylation of MFN2 by SIRT1 (Figures $6 \mathrm{~d}$ and e). Therefore, these results indicate that deacetylated MFN2 through SIRT1 is functionally active.

MFN2 is required for SIRT1-mediated cytoprotection. We next determined cell death after $\mathrm{I} / \mathrm{R}$ using a genetic suppression of MFN2. Adenoviral delivery of a small hairpin RNA targeting MFN2 (AdshMFN2) significantly decreased MFN2 expression (Figure 7b). Importantly, knockdown of 
a

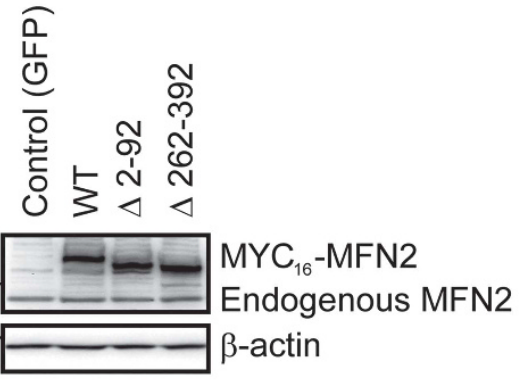

b

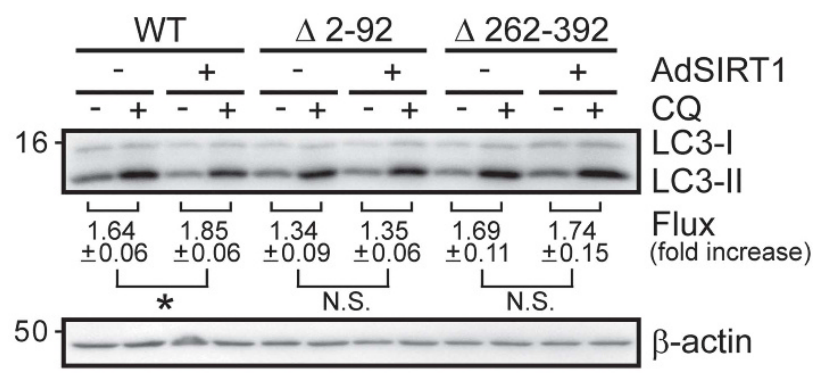

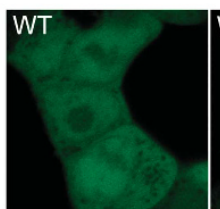
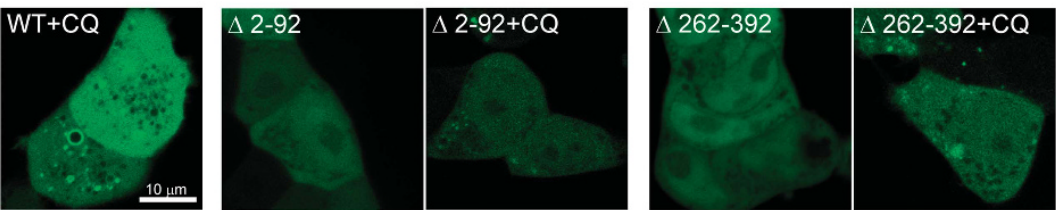

WT +AdSIRT1
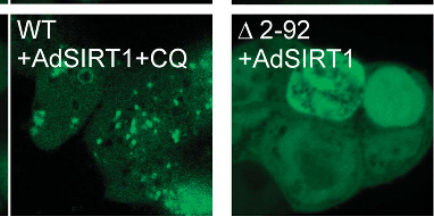

$\Delta$ 2-92

$+A d S I R T 1+C Q$
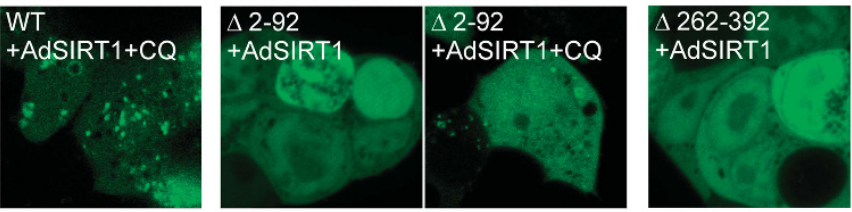

$\triangle 262-392$

+AdSIRT 1 +CQ

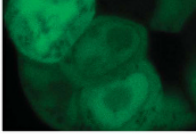

MFN2 KO

e

MFN2 KO

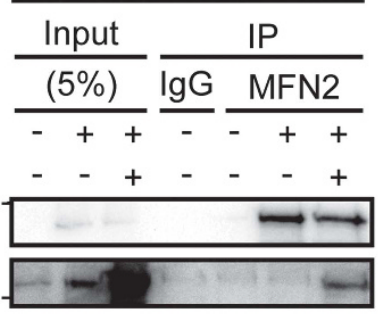

AdMFN2

AdSIRT1

MFN2

\section{SIRT1}
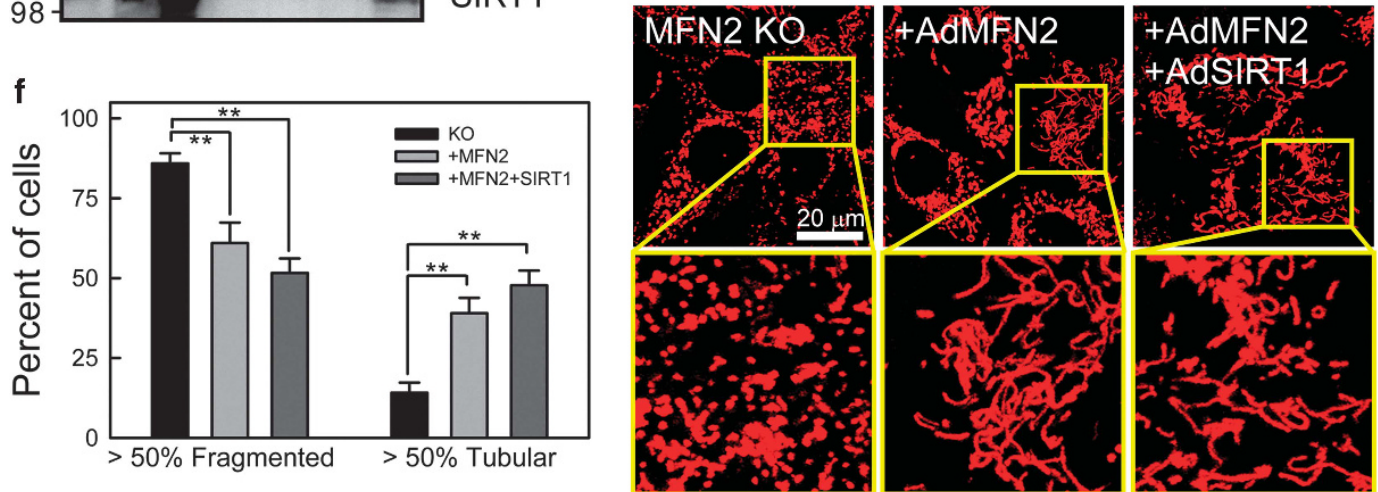

Figure 6 N-terminal deletion of MFN2 abrogates SIRT1-induced autophagy. (a) Validation of MFN2 mutant expression in HEK293T cells by immunoblotting. (b) Densitometric analysis of the LC3-II before and after CQ addition was performed to determine autophagic flux $(n=5)$. ${ }^{*} P<0.05$. NS $=$ nonsignificant. (c) Confocal images of GFP-LC3 with and without CQ. (d) After MFN2 KO MEFs were treated with AdMFN2 alone or both AdMFN2 and AdSIRT1, cell lysates were immunoprecipitated with MFN2, and immunoblotted subsequently with MFN2 or SIRT1. (e) The cell lysates from MFN2 KO MEF were immunoprecipitated with acetyl-K, followed by immunoblotting with MFN2. (f) Confocal images of mitochondrial morphology with TMRM. Bottom panels are magnified images of the square inserts. Mitochondrial morphology was scored by counting individual cell that has either elongated or fragmented mitochondria. ${ }^{* *} P<0.01$

MFN2 abrogated cytoprotection by SIRT1 overexpression, connoting a central role of MFN2 in SIRT1-dependent cytoprotection (Figure 7a). In addition, although hepatocytes overexpressing both scrambled shRNA (control) and SIRT1 retained autophagic flux after $2 \mathrm{~h}$ of reperfusion, silencing of MFN2 abolished SIRT1-induced autophagy after I/R (Figure 7c).
To further understand the role of MFN2 in autophagy, some hepatocytes were subjected to $2 \mathrm{~h}$ of ischemia, a short-term I/R where hepatocytes sustain both autophagy and viability. A significant increase in cell death occurred in shMFN2treated cells (Figure 7d) without altering the expression of SIRT1 and MFN1 during I/R (Figure 7e). Analysis of LC3-II 
a

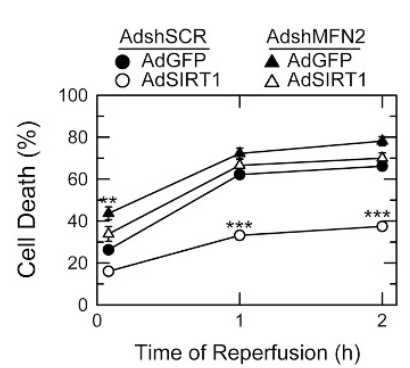

d

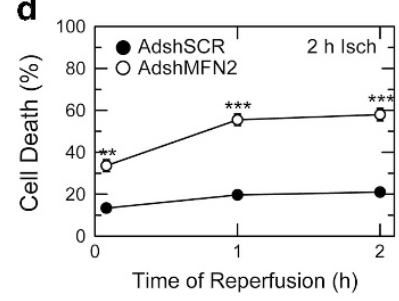

g
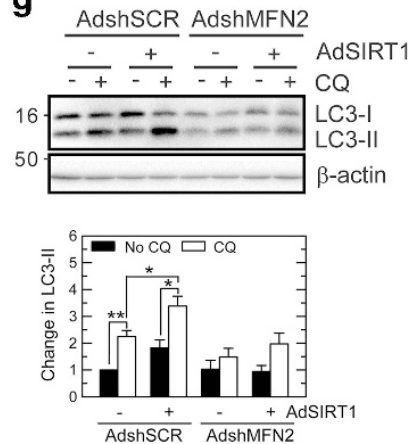

b
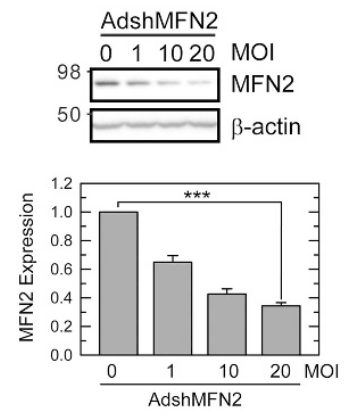

e
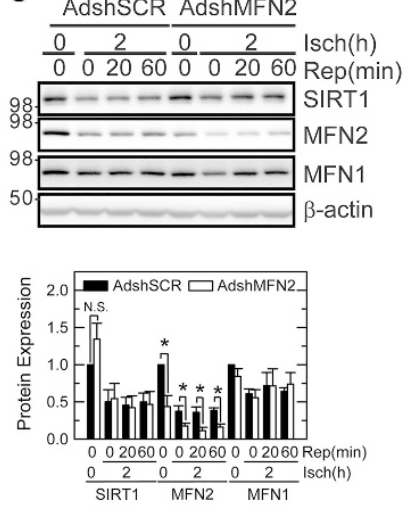

C AdshSCR AdshMFN2
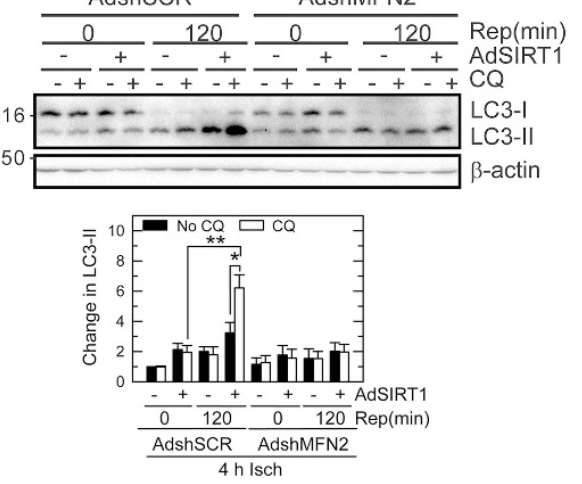

f
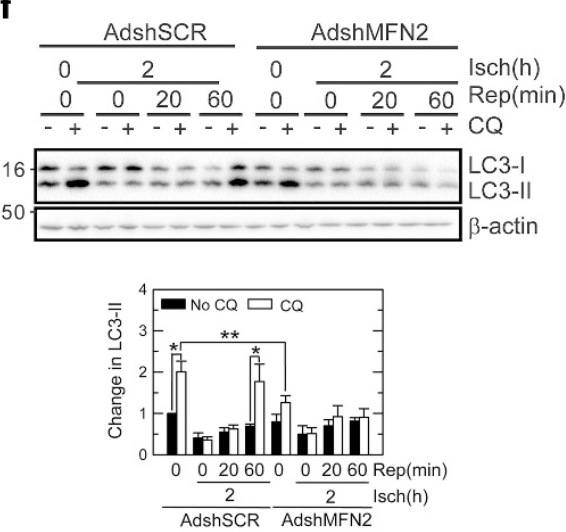

Figure 7 SIRT1-mediated autophagy and cytoprotection requires MFN2. (a) After $4 \mathrm{~h}$ of ischemia, cell death was determined with and without MFN2 knockdown ( $n=3$ ). ${ }^{* *} P<0.01$ and ${ }^{* \star *} P<0.005$ versus AdGFP+AdshSCR. (b) The changes in MFN2 levels with AdshMFN2 $(n=3)$. (c) After $4 \mathrm{~h}$ of ischemia, autophagic flux was analyzed with and without MFN2 knockdown $(n=3)$. (d) The effects of MFN2 knockdown on cell death were determined after $2 \mathrm{~h}$ of ischemia $(n=3)$. (e) After $2 \mathrm{~h}$ of ischemia, the changes in protein levels were assessed with and without MFN2 knockdown $(n=3)$. NS = nonsignificant. The effects of MFN2 knockdown on autophagic flux were evaluated ( $f$ ) after $2 \mathrm{~h}$ of ischemia and $(\mathbf{g})$ during normoxia $(n=3) .{ }^{*} P<0.05$

manifested that silencing of MFN2 halted autophagic flux under this condition (Figure 7f). Basal autophagic flux before I/R was also largely reduced by MFN2 knockdown, ascertaining the importance of MFN2 in basal autophagy as well as in autophagy after I/R. Autophagic flux under normoxia indicated that not only did MFN2 silencing abrogate SIRT1-mediated autophagy, but virtually stalled basal autophagy (Figure $7 \mathrm{~g}$ ), supporting the necessity of MFN2 in basal autophagy. MFN2deficient cells were viable under normoxia (data not shown).

SIRT1 in livers after I/R in vivo. To validate our findings from isolated hepatocytes into in vivo livers, livers were subjected to in vivo I/R with and without SIRT1 overexpression. Immunoblotting demonstrated that reperfusion substantially decreased both SIRT1 and LC3-II levels that was reversed by SIRT1 overexpression (Figures $8 a$ and b). SIRT1 overexpression also significantly increased basal LC3-II before the onset of ischemia, consistent with the results from hepatocytes. Intravital multiphoton images of rhodamine-123 (Rd-123), a $\Delta \Psi_{m}$ indicator, ${ }^{4,5}$ indicated improved membrane potential by SIRT1 (Figure 8c).
Whereas most Rd-123 fluorescence disappeared in control livers after reperfusion, SIRT1-overexpressed livers maintained punctate, green fluorescence of $\mathrm{Rd}-123$ in hepatocytes, indicative of polarized mitochondria. Multiphoton imaging of mCherry-GFP-LC3 displayed that SIRT1 overexpression considerably increased both yellow (autophagosomes) and red (autolysosomes) LC3 puncta in normoxic livers, confirming enhanced basal autophagy by SIRT1 (Figure 8d). Of importance, impaired autophagy in control livers was not observed in SIRT1-overexpressed livers (Figure $8 \mathrm{e}$ ). Thus, in vivo results not only confirm the findings from hepatocytes, but also strongly support our conclusion that SIRT1 suppresses impaired autophagy and mitochondrial dysfunction after l/R.

\section{Discussion}

Hepatic $\mathrm{I} / \mathrm{R}$ injury is an acute stress inciting mortality and morbidity of patients undergoing hepatectomy and transplantation. In this study, using both human and mouse livers, we demonstrate that $\mathrm{I} / \mathrm{R}$ depletes hepatocytes of SIRT1 that sequentially induces autophagic and mitochondrial dysfunction 
a
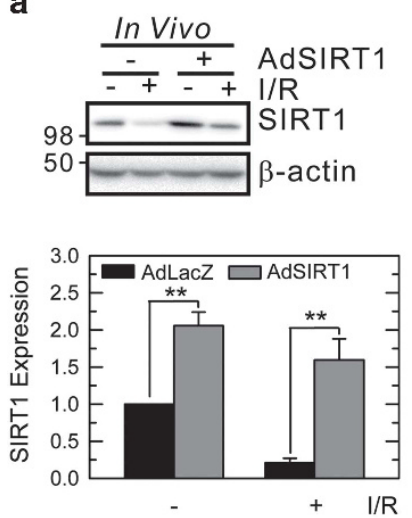

b
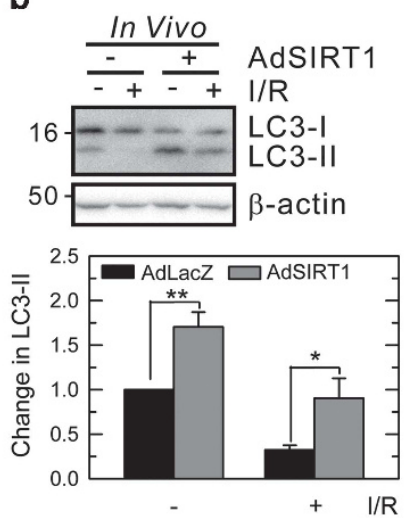

d
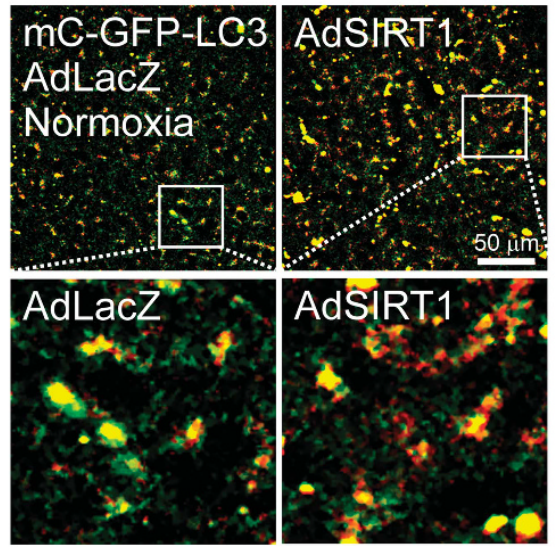

C
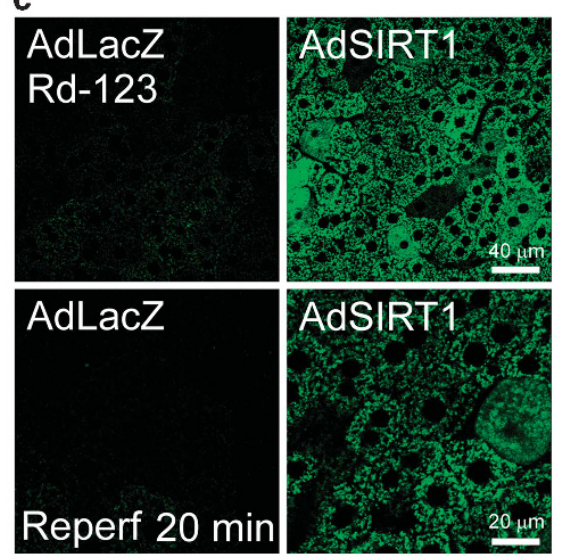

e

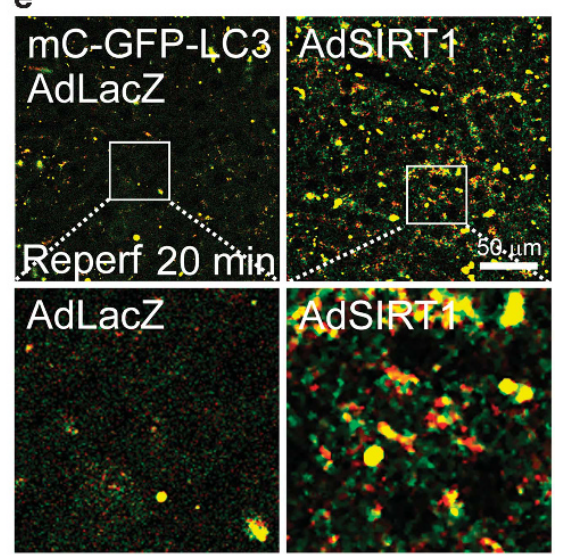

Figure 8 SIRT1 overexpression suppresses mitochondrial and autophagic dysfunction after I/R in vivo in mouse livers. (a) SIRT1 and (b) LC3 were immunoblotted after 20 min of reperfusion with and without SIRT1 overexpression $(n=3)$. ${ }^{*} P<0.05$ and ${ }^{* \star} P<0.01$. (c) Multiphoton images of Rd-123 were collected after reperfusion. The bottom panels are twice enlarged images of the top panels. Multiphoton microscopy of mCherry-GFP-LC3 was conducted (d) during normoxia and (e) after reperfusion. Yellow and red puncta represent autophagosomes and autolysosomes, respectively. The bottom panels represent magnified images of the square inserts at the top panels

and, ultimately, liver failure after reperfusion. Genetic and pharmacological enhancement of SIRT1 confers cytoprotection against I/R through upregulation of autophagy, substantiating the importance of autophagy in hepatic reperfusion injury. In addition, we identify MFN2 as a new mitochondrial target for SIRT1.

As a critical catabolic pathway for both maintaining normal cellular functions and preventing the development of pathological states, autophagy determines cellular fate following hepatic I/R injury. Cells deploy autophagy as an adaptive strategy against diverse stresses. For instance, the induction of autophagy is often observed early after an inciting insult. $^{4-6,20,21}$ In some cases such as I/R injury, stress itself directly impairs autophagy via modifying key autophagyrelated proteins. We have shown that calpain activation after prolonged I/R degrades ATG7 and BECN1 in hepatocytes, precipitating in defective autophagy, onset of the MPT, failure of mitochondrial bioenergetics, and eventually cell death. ${ }^{4,6}$

SIRT1 is present in the extramitochondrial compartments. A major finding here is that cytosolic SIRT1 is indispensable to sustaining functional and structural integrity of the mitochondria after I/R (Figure 2). Although hepatocytes from SIRT1 conditional KO mice showed no signs of mitochondrial dysfunction at basal conditions, they developed a rapid onset of MPT and mitochondrial depolarization even after a shortterm I/R, a condition inducing only minimal injury to WT cells. Hence, the adverse impact on the mitochondria by SIRT1 deletion likely becomes manifest under stresses. This view is consistent with the report that SIRT1-null mice are born alive but are more sensitive to insults than WT animals. ${ }^{22}$ Similarly, SIRT1-KO hepatocytes exhibit no phenotypic abnormalities under normal conditions. ${ }^{23}$ Based on our observation of autophagic and mitochondrial dysfunction after SIRT1 loss, SIRT1 appears to facilitate clearance of dysfunctional or damaged mitochondria. During normoxia, the number of damaged mitochondria is few so that the demand for mitophagy would be minimal. However, reperfused mitochondria suffer ample injury and those injured mitochondria should be eliminated through mitophagy for hepatocytes to remain viable. Though SIRT1 might be involved in yet unknown and autophagy-independent mechanisms, our results support that SIRT1 induces mitophagy, leading to attenuation of MPTmediated mitochondrial dysfunction and subsequent cell injury.

It appears that the N-terminus of MFN2, especially two domains proximal to the GTPase domain (2-92 and 262-392), is critical in SIRT1-mediated autophagy induction (Figure 6). A recent work proposes that SIRT1 deacetylates Lys residues within either $\mathrm{X} 6-\mathrm{K}-[\mathrm{Y}, \mathrm{W}, \mathrm{F}]-\mathrm{X} 5$ or $\mathrm{X} 6-\mathrm{K}-\mathrm{X} 5-[\mathrm{Y}, \mathrm{W}, \mathrm{F}]$ motif. $^{24}$ 
Our bioinformatic analysis conforming to these specific sequences predicts at least five different SIRT1 target sites of MFN2: K37, K215, K357, K655, and K662. Our results with mutant constructs suggest that both K37 and K357 are likely involved in autophagy induction by SIRT1. Noticeably, K215 localizes at the GTPase domain, a critical site for catalytic activity of MFN2. Both $\mathrm{K} 655$ and $\mathrm{K} 662$ reside in the C-terminal flanking domain (604-757) that governs mitochondrial localization of MFN2. ${ }^{18}$ Future studies are warranted to characterize how these residues affect MFN2/SIRT1 interaction.

Approximately $35 \%$ of mitochondrial proteins are acetylated, ${ }^{25} 24 \%$ of which are further associated with energy homeostasis. ${ }^{26}$ It is noteworthy that obesity and hepatic steatosis, diseases closely associated with mitochondrial and autophagic dysfunction, ${ }^{27}$ are mechanistically linked to hyperacetylation of mitochondrial proteins. ${ }^{28,29}$ Here we provide evidence that removing acetyl group of MFN2 by SIRT1 plays a pivotal role in hepatocyte autophagy and survival after I/R injury. Although SIRT1 does not alter the GTPase activity of MFN2, SIRT1 might influence other function of MFN2, including tethering the mitochondria to the endoplasmic reticulum and modulating calcium homeostasis. ${ }^{30}$ Despite a structural similarity, it seems that MFN 1 and 2 are not functionally redundant. ${ }^{30}$

SIRT1 markedly decreased at the protein level after I/R. However, there were no changes in SIRT1 mRNA, implying the presence of distinct post-translational mechanism(s) accounting for SIRT1 depletion. This view is supported by previous studies that SIRT1 protein levels, but not its mRNA, decrease by various stresses. ${ }^{31,32}$ We have shown that activation of calpains causes impaired autophagy, mitochondrial dysfunction, and hepatocyte death after I/R. ${ }^{4-6}$ As calpain inhibition suppressed both SIRT1 loss and cell death after I/R, calpains are, at least in part, an integral contributor to SIRT1 loss. The causative roles of calpains in autophagy defects have also been reported in obesity. ${ }^{33}$ However, it is still important to note that factors other than calpains may attribute to SIRT1 loss. Identification of other factors remains to be elucidated.

An intriguing observation here is that although hepatocytes were tolerant of a large loss in SIRT1, the cells experiencing a similar reduction of MFN2 underwent more severe necrosis after reperfusion (Figure 7), suggesting a central role of MFN2 in $\mathrm{I} / \mathrm{R}$ injury. Increased autophagy defects, mitochondrial dysfunction, and cell death have been found in MFN2-null cardiomyocytes after I/R. ${ }^{34}$ MFN2 KO animals are embryonically lethal, ${ }^{35}$ whereas SIRT1 KO mice are born alive. Thus, it is plausible that MFN2-deficient cells may be more prone to I/R injury than SIRT1-null cells. Future studies are required to determine the minimal levels of MFN2 needed for adequate responses to $\mathrm{I} / \mathrm{R}$ and other stresses. Nevertheless, our findings underscore the importance of both SIRT1 and MFN2 in the orchestrated regulation of autophagy and cell survival after I/R.

In conclusion, prolonged I/R depletes hepatocytes of SIRT1 that in turn leads to a temporal sequence of defective autophagy, mitochondrial dysfunction, and cell death. SIRT1-mediated cytoprotection requires its interaction with MFN2 and subsequent deacetylation of this mitochondrial protein. Inhibition of SIRT1 depletion could be a new therapeutic intervention to improve hepatic function after liver resection or transplantation.

\section{Materials and Methods}

Human liver tissue. Human liver tissue was collected, stored, and treated according to the protocol approved by the institutional review board at the University of Florida. Liver tissue was harvested before and after $15 \mathrm{~min}$ of inflow occlusion, and frozen immediately in liquid nitrogen.

Primary mouse hepatocytes. All animals received humane care according to protocols approved by the institutional care and use committee at the University of Florida. Hepatocytes were isolated from 3-month-old male $\mathrm{C} 57 \mathrm{BL} / 6$ mice by the collagenase perfusion method, as previously described. ${ }^{5}$ Only hepatocytes with viability $>90 \%$, as judged by Trypan blue exclusion, were used. Hepatocytes were plated in Waymouth medium (Life Technologies, Grand Island, NY, USA) supplemented with $10 \%$ fetal bovine serum, $100 \mathrm{nM}$ insulin, and $100 \mathrm{nM}$ dexamethasone and pencillin/streptomycin.

In vitro I/R. Simulated I/R was performed as previously described. ${ }^{4}$ The anoxic buffer consisting of Krebs-Ringer-HEPES (KRH) buffer ( $15 \mathrm{mM} \mathrm{NaCl}, 5 \mathrm{mM} \mathrm{KCl}$, $2 \mathrm{mM} \mathrm{CaCl}_{2}, 1.2 \mathrm{mM} \mathrm{MgSO}_{4}, 1 \mathrm{mM} \mathrm{KH}_{2} \mathrm{PO}_{4}$, and $25 \mathrm{mM} \mathrm{HEPES}$, at pH 6.2) was equilibrated in an anaerobic chamber (Coy Laboratory Products, Ann Arbor, Ml, USA) overnight. To stimulate ischemia, cells were incubated with anoxic $\mathrm{KRH}$ at $\mathrm{pH}$ 6.2 in the anaerobic chamber for up to $4 \mathrm{~h}$. To simulate reoxygenation and return to physiologic $\mathrm{pH}$ during reperfusion, anaerobic $\mathrm{KRH}$ at $\mathrm{pH} 6.2$ was replaced with aerobic $\mathrm{KRH}$ at $\mathrm{pH} 7.4$.

In vivo I/R. Hepatic ischemia in male mice was achieved by occluding the portal triad for $45 \mathrm{~min}$ as previously described. ${ }^{5}$ Reperfusion was initiated by removing the microvascular clamp. Liver biopsies from the left lateral lobe were collected before and during $\mathrm{I} / \mathrm{R}$ and snap-frozen into liquid nitrogen for biochemical analyses.

SIRT1 conditional KO mice. Mice with floxed SIRT1 alleles (SIRT1 ${ }^{\mathrm{F} / \mathrm{FI}}$ ) harboring a Cre-ERT recombinase fused to a triple mutant form of the human estrogen receptor were provided by Dr. Sinclair. ${ }^{12}$ To induce SIRT1 deletion, male $\mathrm{SIRT} 1^{\mathrm{F} / \mathrm{Fl} \text {; }}$ Cre-positive mice were given two consecutive intraperitoneal injections of tamoxifen $(100 \mu \mathrm{l})$ dissolved in a sterile corn oil $(40 \mathrm{mg} / \mathrm{ml}) 3$ days apart. Livers were analyzed after $48 \mathrm{~h}$ following the second tamoxifen injection. Genotyping for SIRT1 and Cre were performed by PCR from ear punch or tail snip biopsies. Genomic DNA was isolated by digesting tissue in an alkaline lysis buffer $(50 \mathrm{mM}$ $\mathrm{NaOH}$ and $0.2 \mathrm{mM}$ EDTA) for $30 \mathrm{~min}$ at $95^{\circ} \mathrm{C}$.

RT-PCR. Messenger RNA was extracted from $10^{6}$ hepatocytes using $500 \mu \mathrm{l}$ of Trizol reagent (Invitrogen, Carlsbad, CA, USA) followed by isopropanol precipitation. The concentrations and purity of mRNA were measured using an Eon spectrophotometer (Biotek Instruments, Winooski, VT, USA) with a Take3 microvolume plate (Biotek Instruments). The cDNA was synthesized with $2 \mathrm{ng}$ mRNA using the SuperScript III First-Strand synthesis system (Invitrogen). For RT-PCR, CDNA $(2 \mu \mathrm{l})$ was used with $1 \mu \mathrm{l}$ of $100 \mathrm{nM}$ primers for SIRT1 (forward: $5^{\prime}$-GCCCATTAAAGCAGTATGTG-3' and reverse: 5'-CATGTAATCTCAACCTTGAG-3') and $\beta$-actin (forward: $5^{\prime}$-GTGGGCCGCTCTAGGCACCAA- $3^{\prime}$ and reverse: $5^{\prime}$-CTCT TTGATGTCACGCACGATTTC- $3^{\prime}$ ). PCR was carried out with the OneTaq DNA Polymerase system (New England Bio Labs, Ipswich, MA, USA) and the MasterCycler 5333 Version 2 (Eppendorf, Hamburg, Germany). The cDNA was denatured at $95^{\circ} \mathrm{C}$ for $5 \mathrm{~min}$ followed by 32 cycles of $95^{\circ} \mathrm{C}$ for $30 \mathrm{~s}, 55^{\circ} \mathrm{C}$ for $30 \mathrm{~s}$, and $72{ }^{\circ} \mathrm{C}$ for $1 \mathrm{~min}$.

Confocal microscopy. Confocal images of TMRM, calcein, PI, GFP-LC3, and mCherry-GFP-LC3 were collected with a gas-tight chamber (Zeiss, Jena, Germany) using an inverted Zeiss 510 laser scanning confocal microscope, as previously described. ${ }^{5}$

Intravital multiphoton microscopy.. To visualize polarized mitochondria, autophagosomes, and autophagic flux, livers were labeled with $\mathrm{Rd}-123$, adenoviral GFP-LC3, or mCherry-GFP-LC3, respectively. ${ }^{4-6}$ After 20 min of reperfusion in vivo, the liver was gently withdrawn from the abdominal cavity and placed over a glass coverslip on the stage of a Zeiss LSM510 equipped with a multiphoton microscope (Zeiss). Images of green fluorescing Rd-123 and GFP-LC3 were collected with a 
×40 water-immersion objective lens. Rd-123 and GFP-LC3 were excited with $780 \mathrm{~nm}$ from a Chameleon Ultra Ti-Sapphire pulsed laser (Coherent Inc., Santa Clara, CA, USA) and images were collected through $500-550 \mathrm{~nm}$ band-pass filter. mCherry-GFP-LC3 was excited at $800 \mathrm{~nm}$ and emission was separated through 500-530 nm (GFP) and 565-615 nm (mCherry) band pass filters. Twenty images were randomly collected per liver.

Separation of cytosolic and membrane fraction. Hepatocytes $\left(3.5 \times 10^{6}\right.$ per $100 \mathrm{~mm}$ dish) were washed and scraped using $140 \mu \mathrm{l}$ of cold $\mathrm{KRH}$ (pH 7.4) containing protease and phosphatase inhibitors. Samples were centrifuged at $1000 \times g$ for $5 \mathrm{~min}$ and the resulting supernatant was centrifuged at $21000 \times g$ at $4{ }^{\circ} \mathrm{C}$ for $15 \mathrm{~min}$ from which the supernatant was collected as the cytoplasmic fraction. The pellet from the original $1000 \times g, 5$ min centrifugation was exposed to a freeze-thaw cycle using liquid nitrogen, and then homogenized in $500 \mu \mathrm{l}$ of $10 \mathrm{mM}$ Tris ( $\mathrm{pH} \mathrm{7.6)} \mathrm{with} \mathrm{phosphatase} \mathrm{and} \mathrm{protease} \mathrm{inhibitors.} \mathrm{The} \mathrm{homogenates} \mathrm{were} \mathrm{then}$ centrifuged at $600 \times g$ at $4^{\circ} \mathrm{C}$ for $5 \mathrm{~min}$ to remove unbroken cells. The supernatant was recentrifuged at $14000 \times \mathrm{g}$ at $4^{\circ} \mathrm{C}$ for $10 \mathrm{~min}$ to obtain the total membrane fraction in the pellet that was resuspended in lysis buffer for immunoblotting or immunoprecipitation. For the subcellular fractionation after $4 \mathrm{~h}$ of ischemia, all reagents were acidified to $\mathrm{pH} 6.2$ and the homogenates underwent two additional centrifugations at $600 \times g$ at $4^{\circ} \mathrm{C}$ for $5 \mathrm{~min}$ before membrane separation.

Transmission electron microscopy. Electron micrographs were prepared as previously described. ${ }^{6}$ Briefly, a monolayer of hepatocytes was fixed using deoxygenated or oxygenated $2 \%$ paraformaldehyde and $2 \%$ glutaraldehyde, $125 \mathrm{mM}$ cacodylate, and $2.2 \mathrm{mM} \mathrm{CaCl}_{2}$ at $\mathrm{pH} 6.2$ for ischemia and at $\mathrm{pH} 7.4$ for reperfusion. Cells were fixed overnight and washed in a cacodylate buffer $(100 \mathrm{mM}$ cacodylate, $7 \%$ sucrose at $\mathrm{pH} 7.4$ ) before staining with $1 \% \mathrm{OsO}_{4}$ (Electron Microscope Science, Hatfield, PA, USA). After $1 \mathrm{~h}$ at room temperature, a Michaelis buffer $(0.1 \mathrm{M}$ $\mathrm{HCl}, 25 \%$ sucrose, $2.8 \mathrm{mM}$ sodium acetate $3 \mathrm{H}_{2} \mathrm{O}, 2.8 \mathrm{mM}$ sodium barbiturate) was used to wash the cells before nuclear staining with a Kellenberger buffer $(0.1 \mathrm{M} \mathrm{HCl}$, $2.8 \mathrm{mM}$ sodium acetate $3 \mathrm{H}_{2} \mathrm{O}, 2.8 \mathrm{mM}$ sodium barbiturate, $2 \%$ uranyl acetate). Hepatocytes were then dehydrated using 70,90 and 100\% ethanol gradient for $15 \mathrm{~min}$ intervals before embedding (Embed-812, Electron Microscope Science).

Immunoblotting and antibodies. Immunoblotting was performed as previously described. ${ }^{5}$ The antibodies used were FOX01, MFN1, and MFN2 (Abcam, Cambridge, MA, USA), VDAC, Cox IV, Lamin B, FOXO3A, ATG7, ATG14L, ATG12, ATG3, ATG4B, BECN1, LC3B, Lamin B, phosphorylated AMPK, total AMPK, phosphorylated Akt, total Akt, phosphorylated $p 70^{s 6 \mathrm{k}}$, total $\mathrm{p} 70^{\mathrm{s} 6 \mathrm{k}}$, and acetylated Lysine (Cell Signaling, Danvers, MA, USA), PGC-1 $\alpha$ (Novus Biologicals, Littleton, CO, USA), $\beta$-actin (Sigma Chemical Co., St. Louis, MO, USA), SIRT1 (Millipore, Temecula, CA, USA), $\beta$-tubulin, Cathepsin D, calnexin, and P21 (Santa Cruz Biotechnology, Santa Cruz, CA, USA). Changes in protein expression were determined using Image $\mathrm{J}$ software (National Institutes of Health, Bethesda, MD, USA).

Immunoprecipitation. IP was performed as previously described. ${ }^{5}$ For IP for acetylation, membrane fractions from 3 separate mouse hepatocyte isolations were pooled, and $250 \mu \mathrm{g}$ input was incubated overnight with $0.75 \mu \mathrm{g}$ acetyl-K antibody (ImmuneChem, Burnaby, BC, Canada) and FOXO1 or FOXO3A antibody (Abcam) at $4^{\circ} \mathrm{C}$. Immunoprecipitates were incubated with Protein $\mathrm{A} / \mathrm{G}$ Plus Agarose (Santa Cruz Biotechnology) at $4^{\circ} \mathrm{C}$ for $1 \mathrm{~h}$. Rabbit and mouse immunoglobulin $\mathrm{G}$ (Santa Cruz Biotechnology) were used as a control to assess nonspecific binding. For IP in MFN2-KO MEFs, cells were lysed in ice-cold lysis buffer containing protease and phosphatase inhibitors, and equal protein amounts were incubated with antibodies against $\mathrm{HA}$ (Y-11; Santa Cruz, Sc-805), acetyl-K, or IgG alone at $4^{\circ} \mathrm{C}$ for $24 \mathrm{~h}$. Protein-antibody complexes were precipitated using Protein-Plus A/G Agarose beads for $2 \mathrm{~h}$. Beads were washed 5 times by centrifugation $(1000 \times \mathrm{g}, 5 \mathrm{~min})$ and resuspended in cold lysis buffer after which antibody-protein complexes were eluted by adding $2 \times$ sample buffer and heating at $95^{\circ} \mathrm{C}$ for $5 \mathrm{~min}$. Samples were subsequently analyzed by immunoblotting for MFN2 or SIRT1.

Cell death assay. PI fluorometry was carried out to determine necrotic cell death as previously described. ${ }^{4}$

Autophagy flux. Autophagic flux was measured by treating hepatocytes with $10 \mu \mathrm{M} \mathrm{CQ}$ for $1 \mathrm{~h}$ before ischemia and continuously during I/R. HEK293T cells were incubated for $4 \mathrm{~h}$ in the absence or presence of $20 \mu \mathrm{M} \mathrm{CQ}$. Lysates $(15 \mu \mathrm{g})$ were analyzed on a $15 \%$ acrylamide gel and probed with rabbit polyclonal LC3 antibody (Cell Signaling) followed by monoclonal $\beta$-actin antibody (Sigma Aldrich, St. Louis, MO, USA) as a loading control.

Adenoviral delivery. Adenovirus encoding SIRT1 (AdSIRT1) was provided by Dr. Sadoshima (Rutgers New Jersey Medical School, Newark, NJ, USA). Hepatocytes were infected with AdSIRT1 in a hormonally defined medium. ${ }^{5}$ After $4 \mathrm{~h}$, the medium was replaced with fresh Waymouth medium and incubated overnight. Adenovirus expressing GFP, LacZ, or scrambled shRNA was used as a viral control. For knockdown of MFN2, adenovirus harboring a small hairpin RNA targeting MFN2 (5'-GCTACAGCTCATCATCAGTTA-3') was constructed. For adenoviral delivery in vivo, animals were intraperitoneally injected with $10^{12}$ viral particles of AdSIRT1 or AdGFP.

Mutant construction. QuikChange II mutagenesis kit (Agilent Technologies, Santa Clara, CA, USA) was used to delete amino acids 2-92 or 262-392 in MYCtagged MFN2 (Addgene, plasmid 23213; Cambridge, MA, USA) as the template. Oligonucleotides used for 2-92 deletion were as follows: 5 '-CTTACCAGCT AGAAACGAGatgatgaaggtggcttttttgg- $3^{\prime}$ and $5^{\prime}$-ccaaaaaaagccaccttcatcatCTCGTT TCTAGCTGGTAAG-3'. Oligonucleotides used for 262-392 deletion were as follows: $5^{\prime}$-cctgaacaaccgctgggatatgcgggaagagcggcaagac-3' and $5^{\prime}$-gtcttgccgctcttcccgcatat cccagcggttgttcagg $-3^{\prime}$. The deletion constructs were verified by DNA sequencing.

Cell lines and transfection. MFN2 KO MEFs were cultured as previously reported. ${ }^{19}$ For MFN2 and SIRT1 co-overexpression in KO MEF, cells plated at $10^{5}$ cells $/ \mathrm{cm}^{2}$ were incubated with adenoviral MFN2 (AdMFN2) at $100 \mathrm{MOI}$ in serumfree Dulbecco's modified essential media (DMEM). After $24 \mathrm{~h}$, the media were exchanged to DMEM with $2 \%$ FBS for another $24 \mathrm{~h}$, with or without the addition of AdSIRT1 at $10 \mathrm{MOI}$.

For transfection of HEK293T cells, $2 \times 10^{6}$ cells were plated in $60 \mathrm{~mm}$ plastic dishes. Using serum-free DMEM, MFN2 plasmids were complexed with GenJet Reagent (SignaGen Laboratories, Rockville MD, USA) at a ratio of $1.5 \mu \mathrm{g}$ plasmid DNA $/ 9 \mu$ l GenJet Reagent for $15 \mathrm{~min}$, and then applied to cells. After cells were exposed to MFN2 plasmid complex for $16 \mathrm{~h}$, the media were replaced with fresh DMEM with $10 \% \mathrm{FBS}$, and cells were incubated for additional $24 \mathrm{~h}$ in the absence or presence of AdSIRT1 (2 MOI).

Mitochondria morphology. $2 \times 10^{5}$ cells plated on $20 \mathrm{~mm}$ glass-bottom dishes were treated with AdMFN2 $(100 \mathrm{MOI})$ for $24 \mathrm{~h}$ in serum-free DMEM followed by additional $24 \mathrm{~h}$ in DMEM supplemented with $2 \%$ FBS with or without AdSIRT1 (2 MOI). To visualize the polarized mitochondria, cells were labeled with TMRM $(0.2 \mu \mathrm{M})$ for $30 \mathrm{~min}$. Fluorescence images were obtained using a LSM510 confocal microscope equipped with a humidified chamber maintaining $5 \% \mathrm{CO}_{2}$ and $37^{\circ} \mathrm{C}$. For morphometric analysis, images collected using a $63 x$ oil immersion lens were analyzed by categorizing individual cell as having either predominantly elongated $(>50 \%)$ or fragmented $\left(>50 \%\right.$ ) morphology, similar to the criteria described by Koshiba et al. ${ }^{19}$ For each condition, 60-100 cells were analyzed and repeated at least 3 times.

Materials. All chemicals were purchased from Sigma Aldrich except E-64d (Enzolife, Farmdale, NY, USA), MG-132 (Fischer Scientific, Waltham, MA, USA), ALLM (Calbiochem, Darmstadt, Germany), and SRT1720 (SelleckChem, Houston, TX, USA).

Data analysis. Differences between groups were compared using analysis of variance and post hoc Bonferroni analysis. All experiments are representative of at least three different cell isolations or animals per group. Data represent mean \pm S.E.M.

\section{Conflict of Interest}

The authors declare no conflict of interest.

Acknowledgements. We are grateful to Drs. Junichi Sadoshima (Rutgers New Jersey Medical School), David A. Sinclair (Harvard Medical School), and David C. Chan (Caltech) for providing AdSIRT1, inducible SIRT1 conditional KO mice, and MFN2 KO cells, respectively. This work was supported in part by US National Institutes of Health National Institute of Diabetes and Digestive and Kidney Diseases grant DK079879 and DK090115 (to J-S Kim) and National Institute on Aging AG028740 (to J-S Kim). 
1. Kim J-S, He L, Qian T, Lemasters JJ. Role of the mitochondrial permeability transition in apoptotic and necrotic death after ischemia/reperfusion injury to hepatocytes. Curr Mol Med 2003; 3: 527-535.

2. Kim J-S, He L, Lemasters JJ. Mitochondrial permeability transition: a common pathway to necrosis and apoptosis. Biochem Biophys Res Commun 2003; 304: 463-470.

3. Mizushima N, Levine B, Cuervo AM, Klionsky DJ. Autophagy fights disease through cellular self-digestion. Nature 2008; 451: 1069-1075.

4. Kim J-S, Nitta T, Mohuczy D, O'Malley KA, Moldawer LL, Dunn WA Jr et al. Impaired autophagy: a mechanism of mitochondrial dysfunction in anoxic rat hepatocytes. Hepatology 2008; 47: 1725-1736.

5. Wang JH, Ahn IS, Fischer TD, Byeon Jl, Dunn WA Jr, Behrns KE et al. Autophagy suppresses age-dependent ischemia and reperfusion injury in livers of mice. Gastroenterology 2011; 141: 2188-2199.

6. Kim J-S, Wang JH, Biel TG, Kim DS, Flores-Toro JA, Vijayvargiya R et al. Carbamazepine suppresses calpain-mediated autophagy impairment after ischemia/reperfusion in mouse livers. Toxicol Appl Pharmacol 2013; 273: 600-610.

7. Cohen HY, Miller C, Bitterman KJ, Wall NR, Hekking B, Kessler B et al. Calorie restriction promotes mammalian cell survival by inducing the SIRT1 deacetylase. Science 2004; 305 : 390-392.

8. Rodgers JT, Puigserver P. Fasting-dependent glucose and lipid metabolic response through hepatic sirtuin 1. Proc Natl Acad Sci USA 2007; 104: 12861-12866.

9. Rodgers JT, Lerin C, Haas W, Gygi SP, Spiegelman BM, Puigserver P. Nutrient control of glucose homeostasis through a complex of PGC-1alpha and SIRT1. Nature 2005; 434: 113-118.

10. Marcu R, Wiczer BM, Neeley CK, Hawkins BJ. Mitochondrial matrix $\mathrm{Ca}^{2+}$ accumulation regulates cytosolic NAD+/NADH Metabolism, protein acetylation and sirtuins expression. Mol Cell Biol 2014; 34: 2890-2902.

11. Liu Y, Dentin R, Chen D, Hedrick S, Ravnskjaer K, Schenk S et al. A fasting inducible switch modulates gluconeogenesis via activator/coactivator exchange. Nature 2008; 456: 269-273.

12. Price NL, Gomes AP, Ling AJ, Duarte FV, Martin-Montalvo A, North BJ et al. SIRT1 is required for AMPK activation and the beneficial effects of resveratrol on mitochondrial function. Cell Metab 2012; 15: 675-690.

13. Chen J, Xavier S, Moskowitz-Kassai E, Chen R, Lu CY, Sanduski K et al. Cathepsin cleavage of sirtuin-1 in endothelial progenitor cells mediates stress-induced premature senescence. Am J Pathol 2012; 180: 973-983.

14. Mizushima N, Yoshimori T, Levine B. Methods in mammalian autophagy research. Cell 2010; 140: 313-326.

15. Kimura S, Noda T, Yoshimori T. Dissection of the autophagosome maturation process by a novel reporter protein, tandem fluorescent-tagged LC3. Autophagy 2007; 3: 452-460.

16. Haigis MC, Sinclair DA. Mammalian sirtuins: biological insights and disease relevance. Annu Rev Pathol 2010; 5: 253-295.

17. Nemoto S, Fergusson MM, Finkel T. SIRT1 functionally interacts with the metabolic regulator and transcriptional coactivator PGC-1 $\alpha$. J Biol Chem 2005; 280: 16456-16460.
18. Santel A, Fuller MT. Control of mitochondrial morphology by a human mitofusin. J Cell Sci 2001; 114: 867-874

19. Koshiba T, Detmer SA, Kaiser JT, Chen H, McCaffery JM, Chan DC. Structural basis of mitochondrial tethering by mitofusin complexes. Science 2004; 305: 858-862.

20. Ding WX, Li M, Chen X, Ni HM, Lin CW, Gao W et al. Autophagy reduces acute ethanolinduced hepatotoxicity and steatosis in mice. Gastroenterology 2010; 139: 1740-1752.

21. Ni HM, Bockus A, Boggess N, Jaeschke H, Ding WX. Activation of autophagy protects against acetaminophen-induced hepatotoxicity. Hepatology 2012; 55: 222-232.

22. Cheng HL, Mostoslavsky R, Saito S, Manis JP, Gu Y, Patel P et al. Developmental defects and p53 hyperacetylation in Sir2 homolog (SIRT1)-deficient mice. Proc Natl Acad Sci USA 2003; 100: 10794-10799.

23. Purushotham A, Schug TT, Xu Q, Surapureddi S, Guo X, Li X. Hepatocyte-specific deletion of SIRT1 alters fatty acid metabolism and results in hepatic steatosis and inflammation. Cell Metab 2009; 9: 327-338.

24. Hubbard BP, Gomes AP, Dai H, Li J, Case AW, Considine T et al. Evidence for a common mechanism of SIRT1 regulation by allosteric activators. Science 2013; 339: 1216-1219.

25. Anderson KA, Hirschey MD. Mitochondrial protein acetylation regulates metabolism. Essays Biochem 2012; 52: 23-35.

26. Kim SC, Sprung R, Chen Y, Xu Y, Ball H, Pei J et al. Substrate and functional diversity of lysine acetylation revealed by a proteomics survey. Mol Cell 2006; 23: 607-618.

27. Czaja MJ, Ding WX, Donohue TM, Friedman SL, Kim J-S, Komatsu M et al. Functions of autophagy in normal and diseased liver. Autophagy 2013; 9: 1131-1158.

28. Kendrick AA, Choudhury M, Rahman SM, McCurdy CE, Friederich M, Van Hove JL et al. Fatty liver is associated with reduced SIRT3 activity and mitochondrial protein hyperacetylation. Biochem J 2011; 433: 505-514.

29. Hirschey MD, Shimazu T, Jing E, Grueter CA, Collins AM, Aouizerat B et al. SIRT3 deficiency and mitochondrial protein hyperacetylation accelerate the development of the metabolic syndrome. Mol Cell 2011; 44: 177-190.

30. de Brito OM, Scorrano L. Mitofusin 2 tethers endoplasmic reticulum to mitochondria. Nature 2008; 456: 605-610.

31. Sasaki T, Maier B, Bartke A, Scrable H. Progressive loss of SIRT1 with cell cycle withdrawal. Aging Cell 2006; 5: 413-422.

32. Cordeiro A, de Souza LL, Oliveira LS, Faustino LC, Santiago LA, Bloise FF et al. Thyroid hormone regulation of Sirtuin 1 expression and implications to integrated responses in fasted mice. J Endocrinol 2013; 216: 181-193.

33. Yang L, Li P, Fu S, Calay ES, Hotamisligil GS. Defective hepatic autophagy in obesity promotes ER stress and causes insulin resistance. Cell Metab 2010; 11: 467-478.

34. Zhao T, Huang $X$, Han L, Wang $X$, Cheng $H$, Zhao $Y$ et al. Central role of mitofusin 2 in autophagosome-lysosome fusion in cardiomyocytes. J Biol Chem 2012; 287: 23615-23625.

35. Chen H, Detmer SA, Ewald AJ, Griffin EE, Fraser SE, Chan DC. Mitofusins Mfn1 and Mfn2 coordinately regulate mitochondrial fusion and are essential for embryonic development. J Cell Biol 2003; 160: 189-200.

Supplementary Information accompanies this paper on Cell Death and Differentiation website (http://www.nature.com/cdd) 